\title{
Electron Acceleration in Direct Laser-Solid Interactions Far Beyond the Ponderomotive Limit
}

\author{
Meng Wen,,${ }^{1,2, *}$ Yousef I. Salamin, ${ }^{1,3}$ and Christoph H. Keitel ${ }^{1}$ \\ ${ }^{1}$ Max-Planck-Institute for Nuclear Physics, Saupfercheckweg 1, 69117 Heidelberg, Germany \\ ${ }^{2}$ Department of Physics, Hubei University, Wuhan 430062, China \\ ${ }^{3}$ Department of Physics, American University of Sharjah, POB 26666, Sharjah, United Arab Emirates
}

(Received 19 October 2018; revised manuscript received 5 January 2020; accepted 8 January 2020; published 2 March 2020)

\begin{abstract}
In laser-solid interactions, electrons may be generated and subsequently accelerated to energies of the order of magnitude of the ponderomotive limit, with the underlying process dominated by direct laser acceleration. The breaking of this limit, realized here by a radially polarized laser pulse incident upon a wire target, can be associated with several novel effects. Three-dimensional particle-in-cell simulations show that a relativistic intense laser pulse can extract electrons from the wire and inject them into the accelerating field. Antidephasing, resulting from collective plasma effects, is shown here to enhance the accelerated electron energy by 2 orders of magnitude compared to the ponderomotive limit. It is demonstrated that ultrashort radially polarized pulses produce superponderomotive electrons more efficiently than pulses of the linear and circular polarization varieties.
\end{abstract}

DOI: 10.1103/PhysRevApplied.13.034001

\section{INTRODUCTION}

The generation of energetic electrons by laser interaction with matter has witnessed considerable development over the past four decades. In the interaction of a laser pulse with an underdense plasma (ambient electron density $n_{e} \leq 0.03 n_{c}$, where $n_{c}$ is the critical density [1]), gigaelectronvolt-energy electron beams can be obtained via wakefield acceleration [2]. Larger numbers of electrons can be accelerated in denser plasmas $\left(n_{e}>0.1 n_{c}\right)$ where direct laser acceleration is dominant, to energies below the ponderomotive limit [3-6]. The term ponderomotive limit refers to the maximum energy gain, $m c^{2} a_{0}^{2} / 2$, by a free electron during interaction with a plane-wave laser pulse $[7,8]$. Here, $a_{0}=e E_{0} /(m c \omega)$ is a dimensionless intensity parameter, in which $E_{0}$ is the electric field amplitude and $\omega$ its frequency, while $m$ and $-e$ are the mass and charge, respectively, of the electron and $c$ is the speed of light in vacuum. The ponderomotive scaling [3-8] is widely quoted within the context of discussions of direct laser acceleration [9-11] in the interaction of intense laser fields with dense matter $\left(n_{e}>n_{c}\right)$, including solid-density plasmas. Generation of electrons which gain over seven times

\footnotetext{
*wenmeng@hubu.edu.cn

Published by the American Physical Society under the terms of the Creative Commons Attribution 4.0 International license. Further distribution of this work must maintain attribution to the author(s) and the published article's title, journal citation, and DOI. Open access publication funded by the Max Planck Society.
}

the ponderomotive energy in near-critical plasmas $\left(n_{e} \lesssim\right.$ $n_{c}$ ) has recently been reported [12-21]. This process is associated with important effects, such as the enhancement of ion acceleration in the near-critical plasmas [22].

Electron energy beyond the ponderomotive limit is achieved by antidephasing acceleration (ADA). Electrons reach such energies in near-critical plasmas by selfinjection into the acceleration phase of the laser field, aided by stochastic motion [13,14], transverse electric fields [18,21], and longitudinal electric [15-17] or magnetic fields $[12,20]$. In the absence of antidephasing, the energies of the electrons generated in laser-solid interactions hover, generally, around the ponderomotive limit [3-6]. Only megaelectronvolt electrons are generated when linearly polarized (LP) and circularly polarized (CP) terawatt laser pulses are used. However, an experiment in which a microwire, used as an advanced solid target to generate and transport hot electrons over millimeters [23-25], has recently demonstrated reaching several times the ponderomotive energy, when LP laser pulses are used [26,27]. Near-critical plasmas can be generated in the vicinity of solid targets for some applications [16-18]. Here, our main result ADA is put forward as an extremely efficient mechanism for electron acceleration directly from a solid target. It promises to deliver significantly higher electron energies and to simplify experimental implementation. Previous work on applications associated with electron acceleration to ponderomotive energies, from laser-solid interactions, include ion acceleration [28,29], fast ignition [30], laser hole-boring [31], high-order harmonic generation [32,33], 
half-cycle extreme-ultraviolet pulse generation [34], bremsstrahlung x-ray generation [35], and the generation of terahertz radiation [36]. Progress in these applications stands to be advanced by the availability of more energetic, shorter, and denser electron bunches.

In this paper, the mechanism of ADA in the interaction of radially polarized (RP) laser pulses of ultrashort duration with solid wires is conceived, intuitively explained, and backed up by particle-in-cell (PIC) simulations. Our investigations demonstrate exceptional attosecond picocoulomb electron bunches with energies around $100 \mathrm{MeV}$, i.e., 2 orders of magnitude higher than the ponderomotive limit. In particular, employing RP pulses, electrons are extracted from a wire target and accelerated by the laser fields (for a schematic, see Fig. 1). Self-injection with a small dephasing rate is caused by the collective motion of the plasma electrons and the complex laser-field variations.

\section{MECHANISM OF ANTIDEPHASING ACCELERATION}

Direct laser acceleration by LP pulses leads to the generation of periodic electron bunches [37] and $\mathrm{CP}$ pulses generate spiral currents [25]. In these cases, the azimuthal electron motion [20,38] makes it difficult to define an acceleration phase. By contrast, the acceleration phase in an RP laser field is determined directly by the regions of negative axial electric fields, with their troughs at phases $(2 N-1 / 2) \pi$, where $N$ is an integer [39]. The radial and

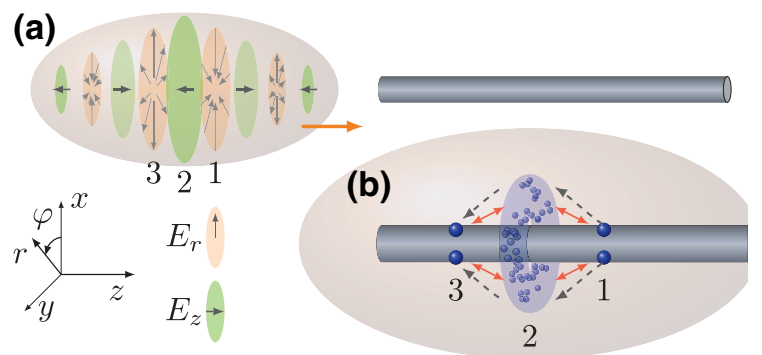

FIG. 1. A schematic of electron generation from a solid wire target, driven by an RP laser pulse. (a) The pulse, with only its oscillating $E_{r}$ and $E_{z}$ shown, is incident upon the left end of the target wire. Troughs of $E_{r}$ and $E_{z}$ and an instantaneous peak of $E_{r}$ are shown at phases 1, 2, and 3, respectively. (b) As the pulse propagates along the wire, electrons are typically pulled out of the target, by the negative $E_{r}$ in phase 1, and subsequently enter phase 2 , where they get accelerated by the negative $E_{z}$. Periodic annular electron bunches are formed at troughs of $E_{z}$, losing some electrons to phase 3 and getting fed by others from phase 1 . Electrons that dephase into phase 3 get pushed back toward the wire by positive $E_{r}$. The dashed black arrows show the trajectories of electrons in the moving frame of the laser pulse and the red arrows represent the repulsion between electrons in different phases (which yields the reduction in dephasing described in the main text). axial electric field components $\left(E_{r}\right.$ and $\left.E_{z}\right)$ of the unperturbed laser pulse are shown schematically in Fig. 1(a). When the RP laser pulse propagates along the wire of radius smaller than its own waist radius at focus, electrons get knocked out and subsequently accelerated by the laser field. In each laser cycle, distortion to the electron distribution and the process of electron injection can typically be described as follows, with the help of Fig. 1. The force associated with $E_{r}$ pulls electrons into the vacuum around phase 1 and pushes them backward relative to the target over phase 3. Meanwhile, electrons get accelerated by $E_{z}$ around phase 2 of a half-cycle and decelerated during interaction with the subsequent half-cycle. Due to the acceleration around the trough of $E_{z}$, dephasing around phase 3 is weaker than that about phase 1 . As shown schematically in Fig. 1(b), the perturbed electrons are gathered around and move in phase 2 with the troughs of $E_{z}$ in an annular region (see also Fig. 2). As a collective plasma effect, the formation of annular bunches has two advantages. On the one hand, as the laser pulse propagates forward, parts of the gathered electrons dephase to phase 3 and get pushed back to the wire by positive $E_{r}$. During dephasing, the affected electrons repel and help those of the annular bunch to stay in phase 2 . On the other hand, new electrons from phase 1 get preaccelerated by those of the annular bunch. Thus they are launched in the accelerating

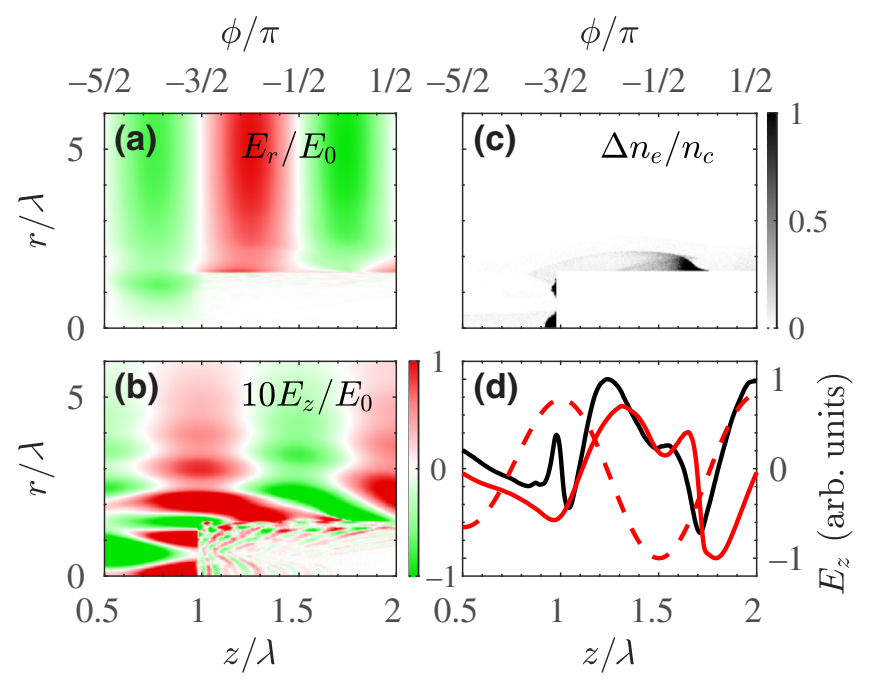

FIG. 2. Snapshots at $t=1.5 \tau$ of (a) $E_{r}$ and (b) $E_{z}$ around the wire target. The electric field components are scaled by the amplitude $E_{0}=E_{r 0}$. The gray shading in (c) corresponds to the electron density perturbation $\Delta n_{e}$. The axial electric field in (b) around the wire is strongly modified by the perturbed electron density. (d) The axial field $E_{z}$ (in arbitrary units) at $r=1.05 r_{0}$ and $r=3 r_{0}$, shown by the solid and dashed red curves, respectively. The solid black line represents the near-wire $E_{z}$ (at $r=$ $1.05 r_{0}$ ) averaged over a laser period, which corresponds to the contribution of the space charge. 
phase with reduced dephasing and injection by ADA is triggered.

\section{PARTICLE-IN-CELL SIMULATIONS}

In our calculations, the wire is assumed to be a cylinder, of length $L$ and radius $r_{0}$, lying with its axis along $+z$, with $z \in[\lambda, \lambda+L]$. The fields of the incident RP pulse are derived from the normalized vector potential, $e \overrightarrow{\mathrm{A}} / m c=a \overrightarrow{\mathrm{e}}_{z}$, where $a$ is given by $[40,41]$

$$
a=\frac{\eta \sqrt{2} a_{0}}{\epsilon \sqrt{1+\alpha^{2}}} \exp \left[\frac{1}{2}-\frac{r^{2}}{w_{0}^{2}} \frac{1-i \alpha}{1+\alpha^{2}}-i \tan ^{-1} \alpha+i \phi\right],
$$

$\overrightarrow{\mathrm{e}}_{z}$ is a unit vector in the direction of $+z$, and $\eta=$ $\operatorname{sech}[(z / c-t) / T]$ replaces the sinc function (encountered in Refs. $[40,41])$ as a temporal pulse envelope, in which $2 \ln (1+\sqrt{2}) T$ is the FWHM of the corresponding intensity profile. In terms of the amplitudes $E_{r 0}$ and $B_{\theta 0}$ of the radial-electric and azimuthal-magnetic field components, the intensity parameter in Eq. (1) is $a_{0}=e E_{r 0} /(m c \omega)=$ $e B_{\theta 0} /(m \omega)$. Also, $\epsilon=w_{0} / z_{r}$, where $w_{0}$ is the waist radius and $z_{r}=\pi w_{0}^{2} / \lambda$ is the Rayleigh length. Furthermore, $\lambda$ is a central wavelength, $\alpha=(z+c t) /\left(2 z_{r}\right)$, and $\phi=\phi_{0}+$ $\omega(z / c-t)$ is the on-axis phase, with $\phi_{0}$ a constant.

Three-dimensional (3D) PIC simulations are conducted, employing the code EPOCH [42], in which a wire target is located in the $20 \times 40 \times 40 \lambda^{3}$ simulation box (moving window) represented by a Cartesian grid of $800 \times$ $800 \times 800$ cells with 200 (5) microparticles for electrons (protons) per cell. The initial target radius, length, and density are given, respectively, by $r_{0}=1.5 \lambda, L=10 \lambda$, and $n_{0}=20 n_{c}$. Target wires of flexible sizes can be made from a number of materials $[23,26,43]$. An ultrashort pulse, with $a_{0}=2, w_{0}=7 \lambda, \lambda=800 \mathrm{~nm}, \phi_{0}=-\pi / 2$, and a duration $T=\tau$ (where $\tau=\lambda / c$ is the laser period), is focused onto the left boundary of the simulation box (at $z=0$ ).

\section{COLLECTIVE EFFECTS OF THE PERTURBED ELECTRONS}

We consider an ultrashort RP laser pulse with $a_{0}=2$ irradiating a wire target. The laser peak arrives at $z=0$ at $t=0$. Snapshots at $t=1.5 \tau$, of the radial and axial electric fields and the perturbation of the electron density $\Delta n_{e}=n_{e}-n_{0}$, are shown in Fig. 2. Over the phase values $\phi \in\left[\phi_{0}, \phi_{0}+\pi\right]$, the radial electric field $E_{r}$ is negative, so it works to knock electrons out of the target wire and the negative $E_{z}$ acts to accelerate them forward, while the opposite happens over the interval $\left[\phi_{0}-\pi, \phi_{0}\right]$. Figure 2(a) shows that the positive $E_{r}$ around the wire is strengthened, due to perturbation of the electrons, while the negative $E_{r}$ is weakened. Figure 2(c) shows that many electrons are collected beyond the cylindrical boundary of the wire, around the phase $\phi_{0}$, at which the unperturbed trough of $E_{z}=-\left|E_{z 0}\right|=\sqrt{2 \exp (1)} \lambda E_{0} /\left(\pi w_{0}\right)$ is expected [44]. The negative electric field gradient, induced by electrons in the annular bunch, shifts the trough of $E_{z}$ to approximately $\phi_{0}+\pi / 2$, as shown in Fig. 2(b), where the trough of $E_{r}$ also sits. The near-wire and off-axis $E_{z}$ are shown by the solid and dashed red curves in Fig. 2(d). While the offaxis $E_{z}$ is unperturbed, the near-wire $E_{z}$ is determined by the time-averaged field, as a contribution from the space charge of the perturbed electrons.

Preacceleration of the electrons in the combined fields around the wire is enhanced by the large surface-to-volume ratio of the wire target, as illustrated in Fig. 3, where snapshots of the accelerating fields are shown at, respectively, times $t=0.5 \tau, 0.75 \tau$, and $\tau$ in (a)-(c). The trajectories of four electrons are shown injected for later acceleration
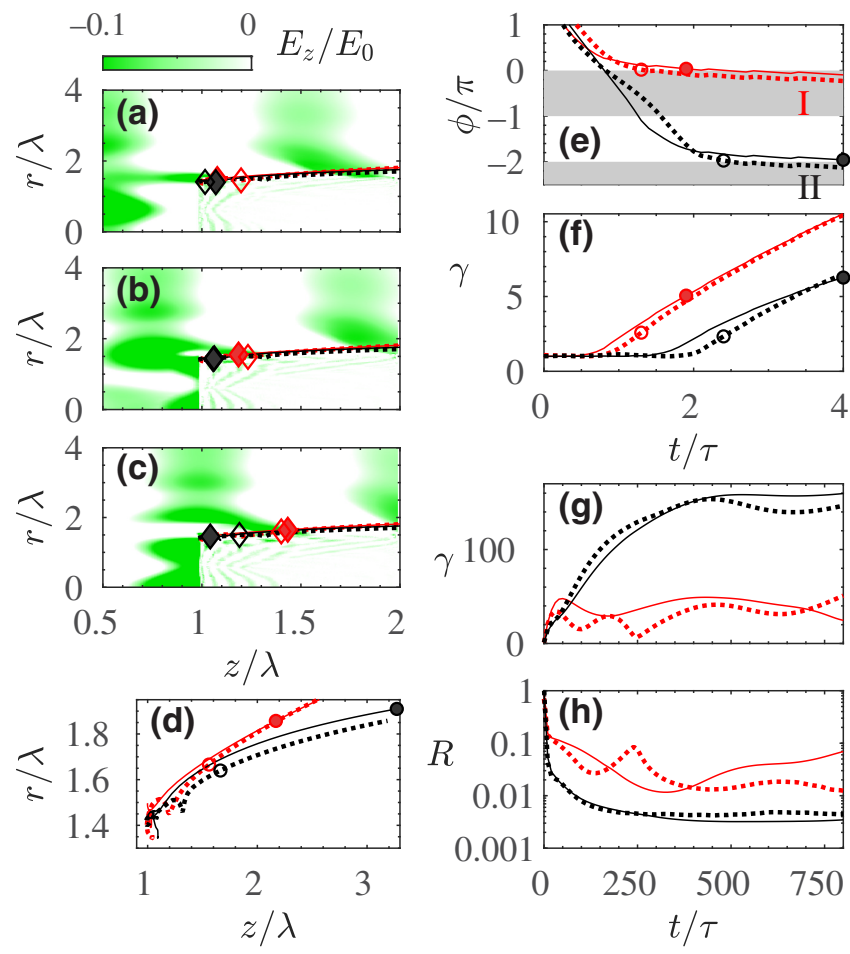

FIG. 3. Density plots in the $r-z$ plane of the accelerating field $\left(E_{z}<0\right)$ are displayed here as snapshots taken at times (a) $0.5 \tau$, (b) $0.75 \tau$, and (c) $\tau$. (d) Trajectories of single electrons that typically originate from the front surface of the wire and stay in the stable phase during acceleration. The same trajectories are also shown in (a)-(c), with their positions at the corresponding times indicated by the diamonds. (e) Variations with time of the phase $\phi$ associated with the electrons considered in (d). The gray-shaded areas represent acceleration phases I $\left[\phi_{0}-\pi / 2, \phi_{0}+\pi / 2\right]$ and II $\left[\phi_{0}-5 \pi / 2, \phi_{0}-3 \pi / 2\right]$, while the circles indicate the time of electrons entering these phases. (f) The evolution in time of the Lorentz factor (or scaled energy) to clearly demonstrate preacceleration of the electrons considered in (d) and (e). The dephasing rates and scaled energies, over longer evolution times, are shown in $(\mathrm{g})$ and $(\mathrm{h})$. 
into different phases. Phase I is centered on $-\pi / 2$ and phase II is around $-5 \pi / 2$. The solid and dashed curves are the trajectories of different particles injected into the same phase and the diamonds in (a)-(c) mark their positions at the corresponding times. Enlargement of the trajectories in Fig. 3(d) reveals that these electrons originate at the front (left) end of the target wire. Prior to entering their respective acceleration phases, the electrons undergo radial and axial oscillations inside the target, forced by the laser field. Subsequently, i.e., when the field structure with forwardshifted negative $E_{z}$ and weakened negative $E_{r}$ is acting and the right phase is reached, they get pulled out of the target slowly and accelerated forward with velocities approaching $c$. Figures 3(e) and 3(f) show the evolution in time of their phases and scaled energies, with the gray shading representing the unperturbed acceleration phase. It is shown, for example, that particles following these typical trajectories can stay in the region with negative $E_{z}$, as they slowly drift in weakened negative $E_{r}$, and can be injected into the accelerating phase with an injection (preacceleration) energy of $\gamma_{0}>1$.

\section{ENERGY GAIN BEYOND THE PONDEROMOTIVE LIMIT}

ADA occurs after the preaccelerated electrons are injected into the laser fields. The dephasing rate is given by $R=-\gamma d \phi /(\omega d t)$, with $R=1$ for an electron initially at rest $[15,18,45]$. Using the relativistic equations of motion, one obtains

$$
\frac{d \gamma}{d \phi}=\frac{p_{z}}{R} \frac{e E_{z}}{m^{2} c^{2} \omega}
$$

where $p_{z}$ is the axial-electron-momentum component and

$$
R=\gamma-\frac{p_{z}}{m c}=1-\frac{e}{m c \omega} \int E_{z} d \phi .
$$

The message of Eq. (3) is quite simple: for an electron initially at rest, $R=1$, but subsequently subjecting the electron to the action of a symmetrically oscillating $E_{z}$ alone will cause $R$ to exhibit symmetric oscillations. However, the laser $E_{z}$, combined with the $E_{z}$ generated by the plasma electrons (especially when comparable to $E_{0} / a_{0}$ [15]) will reduce the dephasing rate significantly.

Figures $3(\mathrm{~g})$ and $3(\mathrm{~h})$ pertain to the electrons during the main acceleration stage. The electrons are launched into that stage by preacceleration with small dephasing rates and get accelerated during a time of approximately $\tau / R$ [46]. Electrons launched into phase II achieve higher energy gains, because the target becomes hotter with time, allowing more electrons to be knocked out and injected. In this regime, stronger space-charge and pinch effects [25] enhance acceleration by the on-axis axial component $E_{z}$ and boost the preacceleration to smaller $R$ [31].
The dephasing rates shown in Fig. 3(h) hover around $R \sim 0.01$ and can be as small as 0.005 . The energy gain receives a boost from the reduction in $R$ via Eq. (2) and approaches approximately $2 \pi e\left|E_{z 0}\right| /(R m c \omega)$. Unfortunately, the optimal antidephasing conditions, which lead to maximum acceleration, cannot be sustained by all electrons. For example, the dephasing rates of electrons accelerated in phase I do not decrease monotonically, as shown in Fig. 3(h), due to the fact that they move radially off axis into regions for which $r>w_{0} / \sqrt{2} \gtrsim r_{0}$, i.e., beyond the position of the peak of $E_{r}$. By contrast, electrons in phase II sustain small $R$ values and acquire higher energy, as shown in Fig. 3(g), which approaches the theoretical limit of $k a_{0} w_{0} \sqrt{2 \exp (1)}$ for vacuum laser acceleration of nanoparticles $[47,48]$.

\section{DISCUSSION}

The self-injection regime of electrons for ADA is discussed through examples involving RP pulses in interaction with wire targets. The RP laser pulse with a discrete accelerating phase, as illustrated schematically in Fig. 1, generates an electron bunch with a FWHM of about 481 as, as shown in Fig. 4(a). More discrete attosecond electron
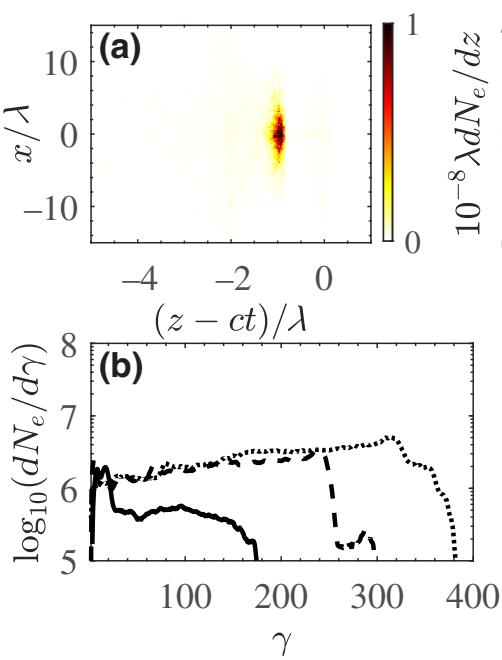
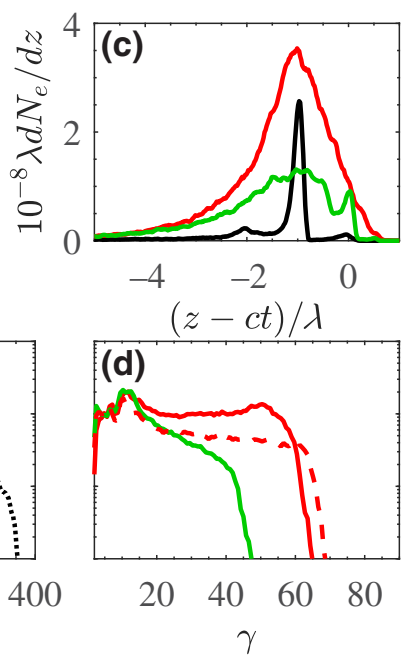

FIG. 4. (a) The space distribution $\left[d N_{e} /(d x d z)\right.$ in arbitrary units] and (b) the energy spectra of electrons driven by RP laser pulses. (c) The axial distributions of electrons driven by RP, LP, and CP laser pulses with FWHMs of 481 attosecond, $5.98 \mathrm{fs}$, and $4.01 \mathrm{fs}$, and beam charges of $15 \mathrm{pC}, 48 \mathrm{pC}$, and $100 \mathrm{pC}$, respectively. (d) The energy spectra of electrons driven by LP and CP pulses. The parameters in (a) and for the solid lines are same as those in Fig. $3\left(a_{0}=2\right.$ and $\left.n_{0}=20 n_{c}\right)$. The $\gamma$ cutoff values of the solid lines in (b) and (d) are 174, 48, and 64, respectively. The black curves represent results driven by RP pulses at $t=450 \tau$. The green and red curves represent electrons at $t=150 \tau$ driven by LP and CP pulses, respectively. The dashed and dotted lines in (b) correspond to cases with $a_{0}=3$ and 4, respectively. The dashed curve in (d) corresponds to the case with $n_{0}=100 n_{c}$. 
bunches with superponderomotive energy can be generated with longer driving pulses. The energy spectra of electrons driven by laser pulses of amplitudes $a_{0}=2$ and higher are shown in Fig. 4(b), which shows the evolution of the beam energy and charge with the driving laser intensity. Further PIC simulations show that the cutoff energies depend on $r_{0}$ only weakly and that the results converge as the resolution is increased. Although the accelerating phase is hard to define in LP and CP pulses, electrons are also gathered at azimuthally dependent phases. Therefore, the ADA regime also works with LP and CP pulses, resulting in the electrons getting accelerated by a continuous stable phase. Similarly to the above, 3D-PIC simulations are carried out in which the RP pulses are replaced by LP and CP pulses of the same amplitudes $a_{0}$, focal radii $w_{0}$, and temporal envelopes $\eta$ (note that such intense single-cycle laser pulses have been demonstrated experimentally [49]). Electrons accelerated by pulses of all three polarizations have continuous spectra. The cutoff energies $\left(48 m c^{2}, 64 m c^{2}\right.$, and $174 m c^{2}$, driven by LP, CP, and RP pulses, respectively) are much higher than the ponderomotive energy gain of $a_{0}^{2} m c^{2} / 2$, with $a_{0}=2$. An RP pulse is evidently superior to the CP and LP pulses for generating high-energy electrons. In the RP case, electrons can be launched into discrete phases, as opposed to being injected into continuous phases using the other polarization modes. Thus, the beam charge in the former is smaller than in the latter. The total charges of the electron beams driven by ultrashort pulses, shown in Fig. 4, are of the order of tens of picocoulombs and increase with the laser duration $[25,26]$. The ratio of the beam charge to the laser duration is similar to that in a laser-wire experiment of approximately the same intensity [50]. The LP and CP laser pulses generate electron beams of lengths that can be controlled by the pulse duration. While the beam charge decreases with increasing initial density, as shown by the dashed red line in Fig. 4(d), the energy gain does not get affected drastically, at least for $\mathrm{CP}$ pulses.

\section{ACKNOWLEDGMENTS}

Y.I.S. acknowledges support for this work by the American University of Sharjah through Faculty Research Grant (No. AS1802).

[1] M. C. Downer, R. Zgadzaj, A. Debus, U. Schramm, and M. C. Kaluza, Diagnostics for plasma-based electron accelerators, Rev. Mod. Phys. 90, 035002 (2018).

[2] T. Tajima and J. M. Dawson, Laser Electron Accelerator, Phys. Rev. Lett. 43, 267 (1979).

[3] S. C. Wilks, W. L. Kruer, M. Tabak, and A. B. Langdon, Absorption of Ultra-Intense Laser Pulses, Phys. Rev. Lett. 69, 1383 (1992).
[4] G. Malka, E. Lefebvre, and J. L. Miquel, Experimental Observation of Electrons Accelerated in Vacuum to Relativistic Energies by a High-Intensity Laser, Phys. Rev. Lett. 78, 3314 (1997).

[5] T. Kluge, T. Cowan, A. Debus, U. Schramm, K. Zeil, and M. Bussmann, Electron Temperature Scaling in Laser Interaction with Solids, Phys. Rev. Lett. 107, 205003 (2011).

[6] M. Thévenet, A. Leblanc, S. Kahaly, H. Vincenti, A. Vernier, F. Quéré, and J. Faure, Vacuum laser acceleration of relativistic electrons using plasma mirror injectors, Nat. Phys. 12, 355 (2015).

[7] F. V. Hartemann, S. N. Fochs, G. P. Le Sage, N. C. Luhmann, J. G. Woodworth, M. D. Perry, Y. J. Chen, and A. K. Kerman, Nonlinear ponderomotive scattering of relativistic electrons by an intense laser field at focus, Phys. Rev. E 51, 4833 (1995).

[8] G. V. Stupakov and M. S. Zolotorev, Ponderomotive Laser Acceleration and Focusing in Vacuum for Generation of Attosecond Electron Bunches, Phys. Rev. Lett. 86, 5274 (2001).

[9] Y. I. Salamin and C. H. Keitel, Electron Acceleration by a Tightly Focused Laser Beam, Phys. Rev. Lett. 88, 095005 (2002).

[10] X. Zhang, V. N. Khudik, and G. Shvets, Synergistic LaserWakefield and Direct-Laser Acceleration in the PlasmaBubble Regime, Phys. Rev. Lett. 114, 184801 (2015).

[11] L.-X. Hu, T.-P. Yu, Z.-M. Sheng, J. Vieira, D.-B. Zou, Y. Yin, P. McKenna, and F.-Q. Shao, Attosecond electron bunches from a nanofiber driven by Laguerre-Gaussian laser pulses, Sci. Rep. 8, 7282 (2018).

[12] Y. I. Salamin, F. H. M. Faisal, and C. H. Keitel, Exact analysis of ultrahigh laser-induced acceleration of electrons by cyclotron autoresonance, Phys. Rev. A 62, 053809 (2000).

[13] Z.-M. Sheng, K. Mima, Y. Sentoku, M. S. Jovanović, T. Taguchi, J. Zhang, and J. Meyer-ter Vehn, Stochastic Heating and Acceleration of Electrons in Colliding Laser Fields in Plasma, Phys. Rev. Lett. 88, 055004 (2002).

[14] S. P. D. Mangles, B. R. Walton, M. Tzoufras, Z. Najmudin, R. J. Clarke, A. E. Dangor, R. G. Evans, S. Fritzler, A. Gopal, C. Hernandez-Gomez, W. B. Mori, W. Rozmus, M. Tatarakis, A. G. R. Thomas, F. S. Tsung, M. S. Wei, and K. Krushelnick, Electron Acceleration in Cavitated Channels Formed by a Petawatt Laser in Low-Density Plasma, Phys. Rev. Lett. 94, 245001 (2005).

[15] A. P. L. Robinson, A. V. Arefiev, and D. Neely, Generating "Superponderomotive" Electrons due to a Non-WakeField Interaction Between a Laser Pulse and a Longitudinal Electric Field, Phys. Rev. Lett. 111, 065002 (2013).

[16] J. P. Geindre, R. S. Marjoribanks, and P. Audebert, Electron Vacuum Acceleration in a Regime Beyond Brunel Absorption, Phys. Rev. Lett. 104, 135001 (2010).

[17] N. Zaïm, M. Thévenet, A. Lifschitz, and J. Faure, Relativistic Acceleration of Electrons Injected by a Plasma Mirror into a Radially Polarized Laser Beam, Phys. Rev. Lett. 119, 094801 (2017).

[18] A. Sorokovikova, A. V. Arefiev, C. McGuffey, B. Qiao, A. P. L. Robinson, M. S. Wei, H. S. McLean, and F. N. Beg, Generation of Superponderomotive Electrons in Multipicosecond Interactions of Kilojoule Laser Beams with Solid-Density Plasmas, Phys. Rev. Lett. 116, 155001 (2016). 
[19] M. Wen, Y. I. Salamin, and C. H. Keitel, Electron acceleration by a radially-polarized laser pulse in a plasma micro-channel, Opt. Express 27, 557 (2019).

[20] B. Liu, H. Y. Wang, J. Liu, L. B. Fu, Y. J. Xu, X. Q. Yan, and X. T. He, Generating Overcritical Dense Relativistic Electron Beams via Self-Matching Resonance Acceleration, Phys. Rev. Lett. 110, 045002 (2013).

[21] M. Vranic, R. A. Fonseca, and L. O. Silva, Extremely intense laser-based electron acceleration in a plasma channel, Plasma Phys. Controlled Fusion 60, 034002 (2018).

[22] J. H. Bin, M. Yeung, Z. Gong, H. Y. Wang, C. Kreuzer, M. L. Zhou, M. J. V. Streeter, P. S. Foster, S. Cousens, B. Dromey, J. Meyer-ter Vehn, M. Zepf, and J. Schreiber, Enhanced Laser-Driven Ion Acceleration by Superponderomotive Electrons Generated from Near-Critical-Density Plasma, Phys. Rev. Lett. 120, 074801 (2018).

[23] R. Kodama et al., Plasma devices to guide and collimate a high density of MeV electrons, Nature 432, 1005 (2004).

[24] B. Ramakrishna, S. Kar, A. P. L. Robinson, D. J. Adams, K. Markey, M. N. Quinn, X. H. Yuan, P. McKenna, K. L. Lancaster, J. S. Green, R. H. H. Scott, P. A. Norreys, J. Schreiber, and M. Zepf, Laser-Driven Fast Electron Collimation in Targets with Resistivity Boundary, Phys. Rev. Lett. 105, 135001 (2010).

[25] V. Kaymak, A. Pukhov, V. N. Shlyaptsev, and J. J. Rocca, Nanoscale Ultradense $z$-Pinch Formation from LaserIrradiated Nanowire Arrays, Phys. Rev. Lett. 117, 035004 (2016).

[26] S. Jiang, L. L. Ji, H. Audesirk, K. M. George, J. Snyder, A. Krygier, P. Poole, C. Willis, R. Daskalova, E. Chowdhury, N. S. Lewis, D. W. Schumacher, A. Pukhov, R. R. Freeman, and K. U. Akli, Microengineering Laser Plasma Interactions at Relativistic Intensities, Phys. Rev. Lett. 116, 085002 (2016).

[27] D. E. Cardenas, T. M. Ostermayr, L. Di Lucchio, L. Hofmann, M. F. Kling, P. Gibbon, J. Schreiber, and L. Veisz, Sub-cycle dynamics in relativistic nanoplasma acceleration, Sci. Rep. 9, 7321 (2019).

[28] G. Mourou, T. Tajima, and S. Bulanov, Optics in the relativistic regime, Rev. Mod. Phys. 78, 309 (2006).

[29] Y. Kishimoto, T. Masaki, and T. Tajima, High energy ions and nuclear fusion in laser-cluster interaction, Phys. Plasmas 9, 589 (2002).

[30] M. G. Haines, M. S. Wei, F. N. Beg, and R. B. Stephens, Hot-Electron Temperature and Laser-Light Absorption in Fast Ignition, Phys. Rev. Lett. 102, 045008 (2009).

[31] N. Iwata, S. Kojima, Y. Sentoku, M. Hata, and K. Mima, Plasma density limits for hole boring by intense laser pulses, Nat. Commun. 9, 623 (2018).

[32] Y. Ueshima, Y. Kishimoto, A. Sasaki, and T. Tajima, Laser Larmor $x$-ray radiation from low- $z$ matter, Laser Part. Beams 17, 45 (1999).

[33] S. Corde, K. Ta Phuoc, G. Lambert, R. Fitour, V. Malka, A. Rousse, A. Beck, and E. Lefebvre, Femtosecond x rays from laser-plasma accelerators, Rev. Mod. Phys. 85, 1 (2013).

[34] H.-C. Wu and J. Meyer-ter Vehn, Giant half-cycle attosecond pulses, Nat. Photonics 6, 304 (2012).
[35] Z. M. Sheng, Y. Sentoku, K. Mima, J. Zhang, W. Yu, and J. Meyer-ter Vehn, Angular Distributions of Fast Electrons, Ions, and Bremsstrahlung $\mathrm{x} / \gamma$-rays in Intense Laser Interaction with Solid Targets, Phys. Rev. Lett. 85, 5340 (2000).

[36] H. B. Zhuo, S. J. Zhang, X. H. Li, H. Y. Zhou, X. Z. Li, D. B. Zou, M. Y. Yu, H. C. Wu, Z. M. Sheng, and C. T. Zhou, Terahertz generation from laser-driven ultrafast current propagation along a wire target, Phys. Rev. E 95, 013201 (2017).

[37] N. Naumova, I. Sokolov, J. Nees, A. Maksimchuk, V. Yanovsky, and G. Mourou, Attosecond Electron Bunches, Phys. Rev. Lett. 93, 195003 (2004).

[38] Y. I. Salamin and F. H. M. Faisal, Relativistic free-electron dynamics and light-emission spectra in the simultaneous presence of a superintense laser field and a strong uniform magnetic field, Phys. Rev. A 58, 3221 (1998).

[39] Y. I. Salamin, Net electron energy gain from interaction with a chirped "plane-wave" laser pulse, Phys. Lett. A 376, 2442 (2012).

[40] Y. I. Salamin, Fields and propagation characteristics in vacuum of an ultrashort tightly focused radially polarized laser pulse, Phys. Rev. A 92, 053836 (2015).

[41] Y. I. Salamin, Fields of an ultrashort tightly focused radially polarized laser pulse in a linear response plasma, Phys. Plasmas 24, 103107 (2017).

[42] T. D. Arber, K. Bennett, C. S. Brady, A. Lawrence-Douglas, M. G. Ramsay, N. J. Sircombe, P. Gillies, R. G. Evans, H. Schmitz, A. R. Bell, and C. P. Ridgers, Contemporary particle-in-cell approach to laser-plasma modelling, Plasma Phys. Controlled Fusion 57, 113001 (2015).

[43] I. A. Andriyash, R. Lehe, A. Lifschitz, C. Thaury, J.-M. Rax, K. Krushelnick, and V. Malka, An ultracompact X-ray source based on a laser-plasma undulator, Nat. Commun. 5, 4736 (2014).

[44] Y. I. Salamin, Electron acceleration from rest in vacuum by an axicon Gaussian laser beam, Phys. Rev. A 73, 043402 (2006).

[45] A. V. Arefiev, B. N. Breizman, M. Schollmeier, and V. N. Khudik, Parametric Amplification of Laser-Driven Electron Acceleration in Underdense Plasma, Phys. Rev. Lett. 108, 145004 (2012).

[46] This time duration may be reduced due to the fact that the phase velocity $v_{p}=c\left(\pi w_{0}^{4}+\lambda^{2} z^{2}\right) /\left(\pi w_{0}^{4}+\lambda^{2} z^{2}-\right.$ $\left.\lambda^{2} w_{0}^{2} / 2\right)$ is clearly greater than $c$ for tightly focused lasers.

[47] P.-L. Fortin, M. Piché, and C. Varin, Direct-field electron acceleration with ultrafast radially polarized laser beams: Scaling laws and optimization, J. Phys. B: At. Mol. Opt. Phys. 43, 025401 (2009).

[48] L. J. Wong and F. X. Kärtner, Direct acceleration of an electron in infinite vacuum by a pulsed radially-polarized laser beam, Opt. Express 18, 25035 (2010).

[49] D. E. Rivas et al., Next generation driver for attosecond and laser-plasma physics, Sci. Rep. 7, 5224 (2017).

[50] H. Nakajima, S. Tokita, S. Inoue, M. Hashida, and S. Sakabe, Divergence-Free Transport of Laser-Produced Fast Electrons along a Meter-Long Wire Target, Phys. Rev. Lett. 110, 155001 (2013). 